\title{
A Maximum Entropy Enhancement for a Family of High-Resolution Spectral Estimators
}

\author{
Augusto Ferrante, Michele Pavon, and Mattia Zorzi
}

\begin{abstract}
Structured covariances occurring in spectral analysis, filtering and identification need to be estimated from a finite observation record. The corresponding sample covariance usually fails to possess the required structure. This is the case, for instance, in the Byrnes-Georgiou-Lindquist THREE-like tunable, high-resolution spectral estimators. There, the output covariance $\Sigma$ of a linear filter is needed to initialize the spectral estimation technique. The sample covariance estimate $\hat{\Sigma}$, however, is usually not compatible with the filter. In this paper, we present a new, systematic way to overcome this difficulty. The new estimate $\Sigma_{\circ}$ is obtained by solving an ancillary problem with an entropic-type criterion. Extensive scalar and multivariate simulation shows that this new approach consistently leads to a significant improvement of the spectral estimators performances.
\end{abstract}

Index Terms-Convex optimization, covariance extension, maximum entropy, multivariable spectral estimation.

\section{INTRODUCTION}

$\mathbf{T}$ HE covariance matrix of a vector extracted from a stationary time series has a Toeplitz structure. Yet, the corresponding sample covariance usually does not share this property. It is then necessary to approximate the sample covariance with a Toeplitz matrix. This is just an instance of a class of problems considered in the important paper [1]. Further motivation comes from considering covariances of the output of a linear filter: They must satisfy a certain constraint dictated by the dynamics which the corresponding sample covariances typically do not.

This problem has been posed and discussed by Georgiou in [2]. Its importance stems from the new approach to spectral estimation (THREE) introduced by Byrnes, Georgiou, and Lindquist in [3] and [4] (see also [5]), which may be viewed as a (considerable) generalization of classical maximum entropy methods. This estimator is particularly competitive in the case of short observation records and in detecting spectral lines. In this approach, the output covariance of a bank of filters, representing measurement devices, is used to extract information on the input power spectrum.

Manuscript received June 04, 2010; revised November 16, 2010; accepted June 23, 2011. Date of publication July 14, 2011; date of current version January 27, 2012. This work was supported in part by the Italian Ministry for Education and Research (MIUR) under PRIN grant 20085FFJ2Z "New Algorithms and Applications of System Identification and Adaptive Control." Recommended by Associate Editor T. Zhou.

A. Ferrante and M. Zorzi are with the Dipartimento di Ingegneria dell'Informazione, Università di Padova, via Gradenigo 6/B, 35131 Padova, Italy (e-mail: augusto@dei.unipd.it, zorzimat@ dei.unipd.it).

M. Pavon is with the Dipartimento di Matematica Pura ed Applicata, Università di Padova, via Trieste 63, 35131 Padova, Italy (e-mail: pavon@math.unipd. it).

Digital Object Identifier 10.1109/TAC.2011.2161842
The filters impose certain structures on the output covariance $\Sigma$ which include Toeplitz and Pick matrix as special cases. It is then necessary to approximate the sample covariance $\hat{\Sigma}$ by nonnegative matrices possessing the required structure. The alternative, as is done in most spectral estimation methods based on second-order statistics, is to use directly the sample covariance [6], [7]. As observed in [2, p.137], however, “... the effect of inaccuracies is not well understood and has not been analyzed in any detail - except via simulation studies."

The purpose of this paper is to introduce a new, systematic approach to deal with these "Caudine Forks" of the method. It may be viewed as an attempt to answer the question raised in [2] generalizing [1]. In order to replace $\hat{\Sigma}$ with a covariance matrix $\Sigma_{\circ}$ having the required structure, we set up an ancillary maximum entropy problem. Indeed, the estimated $\hat{\Sigma}$ plays the role of a prior and the structure requirement may be conveniently turned into a linear constraint, see Proposition 3.2 below. The solution of this variational problem is nontrivial. In spite of several analogies with previous optimization problems considered by the Byrnes-Georgiou-Lindquist school, see [3], [4], [8]-[12], and by Ferrante, Pavon and collaborators, [13], [14], there are also differences which require an original analysis.

The paper is organized as follows. In the next section, we outline THREE-type spectral estimation. Section III features a reformulation of the feasibility of the generalized moment problem. In Section IV, the new variational approach is introduced. Section $\mathrm{V}$ features the dual problem. The following section introduces a matricial Newton method. Section VII is devoted to simulation: The performance of our approach in estimating $\Sigma$ is compared to more simple-minded approaches. We also compare the performance of THREE-like spectral estimators when initialized with our estimate of $\Sigma$ versus other estimates.

\section{THREE-TYPE SPECTRAL ESTIMATION}

A THREE-like spectral estimation method is outlined as follows. The collection of sample data $y_{1} \ldots y_{N}$ of a stochastic process $y$ is fed to a suitably designed bank of rational filters $G(z)$. The steady-state covariance matrix $\Sigma$ of the resulting output is then estimated by statistical methods. Only zerothorder covariance lags of the output need to be estimated, ensuring statistical robustness of the method. Finding now an input process compatible with the estimated $\hat{\Sigma}$ and with rational spectrum of prescribed maximum degree turns into a NevanlinnaPick interpolation problem with bounded degree [15], [16]. The latter can be viewed as a generalized moment problem which is advantageously cast in the frame of various convex optimization problems: We mention, in particular, the covariance extension problem and its generalization, see [3], [9], [17]-[20]. These 
problems pose a number of theoretical and computational challenges for which we also refer the reader to [10], [21], [22], and [23].

Besides signal processing, significant applications of this theory are found in modeling and identification [12], [24], [25], $H_{\infty}$ robust control [8], [11], and biomedical engineering [26]. The first, inevitable step in this procedure is to test for feasibility of the generalized moment problem. Generically, with the estimated $\hat{\Sigma}$, the problem is not feasible, i.e., $\hat{\Sigma}$ does not have the structure imposed by $G(z)$. Various ad hoc procedures are then employed to approximate $\hat{\Sigma}$ with another covariance matrix. This step, although this is hardly advertised, is far from harmless. For instance, projecting $\hat{\Sigma}$ onto a suitable subspace (see below) may destroy the definiteness of the matrix.

While our procedure replaces empirical techniques with a precise algorithm, simulation shows that it also leads to a considerable improvement in the estimate of $\Sigma_{\circ}$ in several critical cases, see Section VII. Indeed, there is a significant improvement of the spectral estimator even when the $\Sigma_{\circ}$ obtained by the different methods approximate the true $\Sigma$ with essentially the same level of accuracy! We forecast that this technique will turn out to be useful in different contexts such as multivariate statistics and identification.

\section{Feasibility of the Generalized Moment Problem}

Consider a transfer function

$$
G(z)=(z I-A)^{-1} B, \quad A \in \mathbb{C}^{n \times n}, B \in \mathbb{C}^{n \times m}, n>m
$$

where $A$ has all its eigenvalues in the open unit disk, $B$ has full column rank, and $(A, B)$ is a reachable pair. Suppose $G(z)$ models a bank of filters fed by a wide sense stationary, purely nondeterministic, $\mathbb{C}^{m}$-valued process $y$. Assume that the spectral density $\Phi$ of $y$ is coercive. Let $x$ be the $n$-dimensional stationary output process

$$
x_{k+1}=A x_{k}+B y_{k}, \quad k \in \mathbb{Z} .
$$

We denote by $\Sigma$ the covariance of $x_{k}$. Notice that $\Sigma>0$ since $A$ is a stable matrix, $(A, B)$ is reachable and $\Phi$ is coercive. Let $\mathcal{H}_{n}$ denote the $n^{2}$-dimensional, real vector space of Hermitian matrices of dimension $n \times n$. We denote by $C\left(\mathbb{T}, \mathcal{H}_{m}\right)$ the family of $\mathcal{H}_{m}$-valued, continuous functions on the unit circle $\mathbb{T}$. Let star denote transposition plus conjugation. Consider now the linear operator

$$
\begin{aligned}
\Gamma: C\left(\mathbb{T}, \mathcal{H}_{m}\right) & \rightarrow \mathcal{H}_{n}, \\
\Phi & \mapsto \int G \Phi G^{*}
\end{aligned}
$$

where integration takes place on $\mathbb{T}$ with respect to normalized Lebesgue measure $\mathrm{d} \vartheta / 2 \pi$. It follows that $\Sigma$ belongs to the linear space

$$
\begin{aligned}
\text { Range } \Gamma:=\left\{M \in \mathcal{H}_{n} \mid\right. & \exists \Phi \in C\left(\mathbb{T}, \mathcal{H}_{m}\right) \\
& \text { such that } \left.\int G \Phi G^{*}=M\right\} .
\end{aligned}
$$

In [7], [21] (see also [27]), it was shown that $\Sigma \in \mathcal{H}_{n}$ belongs to Range $\Gamma$ if and only if there exists $H \in \mathbb{C}^{m \times n}$ such that

$$
\Sigma-A \Sigma A^{*}=B H+H^{*} B^{*}
$$

or equivalently if and only if the following rank condition holds:

$$
\operatorname{rank}\left[\begin{array}{cc}
\Sigma-A \Sigma A^{*} & B \\
B^{*} & 0
\end{array}\right]=2 m \text {. }
$$

The dimension of Range $\Gamma$ may now be established along the lines of [10, Lemma 4], which deals with the scalar case, and [2, Page 137], which treats the multivariate real case.

Proposition 3.1: The linear space Range $\Gamma$ has real dimension $m(2 n-m)$.

Proof: The dimension of the linear space Range $\Gamma$ is invariant under a change of basis in the state space of $G$. Since $B$ is assumed to be full column-rank, we can then assume that $B:=$ $\left[\begin{array}{c}I_{m} \\ 0\end{array}\right]$. From (5), we get that dim Range $\Gamma$ equals the real dimension of the linear space of matrices that can be written in the form $B H+H^{*} B^{*}$, or, equivalently (given the structure of B), in the form $\left[\begin{array}{cc}Q & H_{2} \\ H_{2}^{*} & 0\end{array}\right]$, with $Q \in \mathcal{H}_{m}$ and $H_{2} \in \mathbb{C}^{m \times(n-m)}$. Such a dimension is $m(2 n-m)$.

In [13, Proposition 2.1], it was shown that, after normalizing $\Sigma>0$ to the identity matrix, condition (6) could be replaced by a geometric condition. We show next that the latter condition is equivalent to (6) for any Hermitian $\Sigma$.

Proposition 3.2: Given $\Sigma \in \mathcal{H}_{n}$, a necessary and sufficient condition for $\Sigma \in$ Range $\Gamma$ is that the following condition holds:

$$
\left(I-\Pi_{B}\right)\left(\Sigma-A \Sigma A^{*}\right)\left(I-\Pi_{B}\right)=0
$$

where we denote by $\Pi_{B}:=B\left(B^{*} B\right)^{-1} B^{*}$ the orthogonal projection onto Range $B$.

Proof:

Necessity: We know that there exists $H \in \mathbb{C}^{m \times n}$ such that

$$
\Sigma-A \Sigma A^{*}=B H+H^{*} B^{*} .
$$

Pre and post-multiplying this relation by $I-\Pi_{B}$, we obtain

$$
\begin{aligned}
\left(I-\Pi_{B}\right) & \left(\Sigma-A \Sigma A^{*}\right)\left(I-\Pi_{B}\right) \\
= & \left(I-\Pi_{B}\right)\left(B H+H^{*} B^{*}\right)\left(I-\Pi_{B}\right) \\
= & \left(I-\Pi_{B}\right) B H\left(I-\Pi_{B}\right) \\
& +\left[\left(I-\Pi_{B}\right) B H\left(I-\Pi_{B}\right)\right]^{*}=0 .
\end{aligned}
$$

Sufficiency: We exploit condition (6). Let us first consider the matrix

$$
T:=\left[\begin{array}{ll}
C & B
\end{array}\right] \in \mathbb{C}^{n \times n}
$$

where $C \in \mathbb{C}^{n \times(n-m)}$ has full column rank and is such that (Range $C) \perp$ (Range $B$ ), so that $T$ is invertible. Moreover, $C$ can be expressed as $C=\left(I-\Pi_{B}\right) V$, where $V \in \mathbb{C}^{n \times(n-m)}$ has full column rank. In view of (7), we have

$$
C^{*}\left(\Sigma-A \Sigma A^{*}\right) C=0 .
$$


We now consider the matrices

$$
\left[\begin{array}{cc}
\Sigma-A \Sigma A^{*} & B \\
B^{*} & 0
\end{array}\right]
$$

and

$$
\begin{aligned}
\Delta & :=\left[\begin{array}{cc}
T^{*} & 0 \\
0 & I
\end{array}\right]\left[\begin{array}{cc}
\Sigma-A \Sigma A^{*} & B \\
B^{*} & 0
\end{array}\right]\left[\begin{array}{cc}
T & 0 \\
0 & I
\end{array}\right] \\
& =\left[\begin{array}{cc}
T^{*}\left(\Sigma-A \Sigma A^{*}\right) T & T^{*} B \\
B^{*} T & 0
\end{array}\right] .
\end{aligned}
$$

By (9) and (10), we get

$$
\begin{aligned}
\Delta & =\left[\begin{array}{cc|c}
C^{*}\left(\Sigma-A \Sigma A^{*}\right) C & C^{*}\left(\Sigma-A \Sigma A^{*}\right) B & C^{*} B \\
B^{*}\left(\Sigma-A \Sigma A^{*}\right) C & B^{*}\left(\Sigma-A \Sigma A^{*}\right) B & B^{*} B \\
\hline B^{*} C & B^{*} B & 0
\end{array}\right] \\
& =\left[\begin{array}{cc|c}
0 & \star & 0 \\
\star & \star & B^{*} B \\
\hline 0 & B^{*} B & 0
\end{array}\right]
\end{aligned}
$$

where $B^{*} B$ is an invertible matrix, since $B$ has full column rank. Recalling that the rank of a matrix is invariant under multiplication by an invertible matrix, we conclude that

$$
\operatorname{rank}\left[\begin{array}{cc}
\Sigma-A \Sigma A^{*} & B \\
B^{*} & 0
\end{array}\right]=\operatorname{rank} \Delta=2 m
$$

namely, by (6), $\Sigma \in$ Range $\Gamma$.

Consider now the following situation:

- The filter $G(z)$ is fed by the $m$-dimensional data $\left\{y_{i}\right\}_{i=1}^{N}$ and we collect the $n$-dimensional output data $\left\{x_{i}\right\}_{i=1}^{N}$.

- We compute an estimate $\hat{\Sigma}$ of $\Sigma$ in the usual way

$$
\hat{\Sigma}:=\frac{1}{N} \sum_{i=1}^{N} x_{i} x_{i}^{*} .
$$

Notice that $\hat{\Sigma} \in \mathcal{H}_{n}$ and $\hat{\Sigma} \geq 0$. Moreover, for $N \geq n, \hat{\Sigma}$ is positive definite with probability 1 . In general, $\hat{\Sigma}$ does not belong to Range $\Gamma$. Indeed, $m<n$ and Range $\Gamma$ has only dimension $m(2 n-m)<n^{2}$ (Proposition 3.1). We then have to face the following problem: Given $\hat{\Sigma} \in \mathcal{H}_{+, n}:=\left\{\Sigma \in \mathcal{H}_{n} \mid \Sigma>0\right\}$ and $G(z)$ with the previous properties, find a positive definite $\Sigma_{\circ} \in$ Range $\Gamma$ such that $\Sigma_{\circ}$ is, in a suitable sense, as close as possible to $\hat{\Sigma}$. As explained in the introduction, this problem is motivated by THREE-like spectral estimation algorithms, see [4], [5], where an estimate of $\Sigma$ in Range $\Gamma$ is needed to start the algorithm. A simple-minded approach consists in projecting $\hat{\Sigma}$ given by (15) onto Range $\Gamma$ thereby obtaining a new hermitian matrix $\hat{\Sigma}_{\Gamma}$, see e.g., [5, Section 8]. For a large number $N$ of samples, we expect $\hat{\Sigma}_{\Gamma}$ to be close to $\hat{\Sigma}$ since the true state covariance $\Sigma$ does belong to Range $\Gamma$. The projection $\hat{\Sigma}_{\Gamma}$, however, might turn out to be indefinite and this is particularly likely when $N$ is not large. In this case, $\hat{\Sigma}_{\Gamma}$ may be further adjusted by adding to it a matrix of the form $\varepsilon \Sigma_{+}$with $\Sigma_{+} \in \operatorname{Range} \Gamma$, $\Sigma_{+}>0$ and $\varepsilon>0$ so large that

$$
\Sigma_{\circ}=\hat{\Sigma}_{\Gamma}+\varepsilon \Sigma_{+}>0 .
$$

In this way, a positive definite matrix belonging to Range $\Gamma$ is obtained. Notice that a positive definite matrix $\Sigma_{+} \in$ Range $\Gamma$ indeed exists and can be easily computed as follows. Set $H_{+}:=$ $(1 / 2) B^{*}$ and consider the equation

$$
\Sigma_{+}-A \Sigma_{+} A^{*}=B H_{+}+H_{+}^{*} B^{*}=B B^{*} .
$$

Since $(A, B)$ is reachable and $A$ is a stable matrix, we have that (17) admits a unique solution $\Sigma_{+}$and such a solution is indeed positive definite. In view of (5), $\Sigma_{+}$also belongs to Range $\Gamma$.

\section{New ApPROACH to Finding $\Sigma_{\circ}$}

In this section, we present a new systematic procedure to determine a positive definite $\Sigma_{0} \in$ Range $\Gamma$ which is as close as possible to $\hat{\Sigma}$ in a meaningful sense. Recall that a most fundamental (pseudo-)distance in mathematical statistics is the information divergence (Kullback-Leibler index, relative entropy), [28]. For two Gaussian distributions $p_{\Sigma}, p_{\Omega}$ on $\mathbb{R}^{n}$ with zero mean and covariance matrices $\Sigma>0$ and $\Omega>0$, respectively, it is given by

$$
\mathbb{D}\left(p_{\Sigma} \| p_{\Omega}\right):=\frac{1}{2}\left[\log \operatorname{det}\left(\Sigma^{-1} \Omega\right)+\operatorname{tr}\left(\Omega^{-1} \Sigma\right)-n\right] .
$$

Notice that the right-hand side of (18) provides a natural pseudodistance, denoted henceforth by $\mathbb{D}(\Sigma \| \Omega)$, on the space $\mathcal{H}_{+, n}$. This fact leads us to consider the following problem.

Problem 1: Given $\hat{\Sigma} \in \mathcal{H}_{+, n}$ and $G(z)$ as in the previous section, solve

$$
\text { minimize } \mathbb{D}(\Sigma \| \hat{\Sigma}) \text { over } \Sigma \in\left(\mathcal{H}_{+, n} \cap \text { Range } \Gamma\right) .
$$

The solution to Problem 1 provides the required $\Sigma_{\circ}$.

Remark 4.1: In [2], the Umegaki-von Neumann relative entropy [29] was proposed instead, restricting the search to covariances having the same trace as the sample covariance $\hat{\Sigma}$. In alternative, it was there suggested that one could use as distance the one induced by a matrix norm. Our choice is supported by the following considerations. First, as observed in [1, p.963], $\mathbb{D}(\cdot, \cdot)$ "really comes from maximum-likelihood considerations and thus should, in some sense, give us a reasonable answer, even if the process is not Gaussian and the vector samples are not independent". Second, with this distance, the solution turns out to have a simple form and the variational analysis can be carried through to the very end, see below. Finally, simulation shows that THREE-like estimators initialised with $\Sigma_{\circ}$ work extremely well.

In what follows, we assume that $\hat{\Sigma}>0$ and use the compact notation $\Pi_{B}^{\perp}:=I-\Pi_{B}$. In view of Proposition 3.2, we can reformulate Problem 1 as the problem of finding $\Sigma$ in $\mathcal{H}_{+, n}$ minimizing $\mathbb{D}(\Sigma \| \hat{\Sigma})$ subject to the linear constraint

$$
\Pi_{B}^{\perp}\left(\Sigma-A \Sigma A^{*}\right) \Pi_{B}^{\perp}=0 .
$$

Thus, our problem resembles a most standard maximum entropy (or, equivalently, minimum relative entropy) problem [28], [30]. As a first step, we introduce the Lagrangian function

$$
\tilde{L}(\Sigma, \Lambda)=\mathbb{D}(\Sigma \| \hat{\Sigma})+\operatorname{tr}\left[\Lambda \Pi_{B}^{\perp}\left(\Sigma-A \Sigma A^{*}\right) \Pi_{B}^{\perp}\right]
$$




$$
\begin{aligned}
= & -\frac{1}{2} \log \operatorname{det} \Sigma+\frac{1}{2} \log \operatorname{det} \hat{\Sigma}+\frac{1}{2} \operatorname{tr}\left(\hat{\Sigma}^{-1} \Sigma\right) \\
& -\frac{n}{2}+\operatorname{tr}\left[\Lambda \Pi_{B}^{\perp}\left(\Sigma-A \Sigma A^{*}\right) \Pi_{B}^{\perp}\right] .
\end{aligned}
$$

We consider the unconstrained minimization problem

$$
\min _{\Sigma}\left\{\tilde{L}(\Sigma, \Lambda) \mid \Sigma \in \mathcal{H}_{+, n}\right\}
$$

The second and fourth term in the right-hand side of (21) do not depend on $\Sigma$. Hence, it is equivalent to minimize the functional

$$
\begin{aligned}
L(\Sigma, \Lambda)=-\frac{1}{2} \log \operatorname{det} \Sigma & +\frac{1}{2} \operatorname{tr}\left(\hat{\Sigma}^{-1} \Sigma\right) \\
& +\operatorname{tr}\left[\Lambda \Pi_{B}^{\perp}\left(\Sigma-A \Sigma A^{*}\right) \Pi_{B}^{\perp}\right]
\end{aligned}
$$

over $\Sigma \in \mathcal{H}_{+, n}$. The first variation at $\Sigma>0$ in direction $\delta \Sigma \epsilon$ $\mathcal{H}_{n}$ is given by

$$
\begin{aligned}
\delta L(\Sigma, \Lambda, \delta \Sigma) \\
=\lim _{\varepsilon \rightarrow 0} \frac{L(\Sigma+\varepsilon \delta \Sigma, \Lambda)-L(\Sigma, \Lambda)}{\varepsilon} \\
=-\frac{1}{2} \operatorname{tr}\left[\Sigma^{-1} \delta \Sigma\right]+\frac{1}{2} \operatorname{tr}\left[\hat{\Sigma}^{-1} \delta \Sigma\right] \\
\quad+\operatorname{tr}\left[\Lambda \Pi_{B}^{\perp}\left(\delta \Sigma-A \delta \Sigma A^{*}\right) \Pi_{B}^{\perp}\right] \\
=\operatorname{tr}\left[\left(-\frac{1}{2} \Sigma^{-1}+\frac{1}{2} \hat{\Sigma}^{-1}+\Pi_{B}^{\perp} \Lambda \Pi_{B}^{\perp}-A^{*} \Pi_{B}^{\perp} \Lambda \Pi_{B}^{\perp} A\right) \delta \Sigma\right] .
\end{aligned}
$$

By annihilating the first variation for each $\delta \Sigma \in \mathcal{H}_{n}$, we get

$$
\Sigma^{-1}=\hat{\Sigma}^{-1}+2 \Pi_{B}^{\perp} \Lambda \Pi_{B}^{\perp}-2 A^{*} \Pi_{B}^{\perp} \Lambda \Pi_{B}^{\perp} A .
$$

It is then natural to restrict our attention to multiplier matrices $\Lambda \in \mathcal{H}_{n}$ satisfying the inequality

$$
\hat{\Sigma}^{-1}+2 \Pi_{B}^{\perp} \Lambda \Pi_{B}^{\perp}-2 A^{*} \Pi_{B}^{\perp} \Lambda \Pi_{B}^{\perp} A>0 .
$$

For such $\Lambda$, we get that the form of the optimal solution is

$$
\Sigma_{\Lambda}=\left(\hat{\Sigma}^{-1}+2 Q_{\Lambda}\right)^{-1}
$$

where

$$
Q_{\Lambda}:=\Pi_{B}^{\perp} \Lambda \Pi_{B}^{\perp}-A^{*} \Pi_{B}^{\perp} \Lambda \Pi_{B}^{\perp} A .
$$

It is quite interesting to notice that $Q_{\Lambda}$ gives another characterization of Range $\Gamma$ as stated by the following proposition.

Proposition 4.1: Let $\Lambda \in \mathcal{H}_{n}$ and $Q_{\Lambda}$ be defined by (27). Then $Q_{\Lambda} \in$ Range $\Gamma^{\perp}$.

Proof: Let be $\Sigma \in$ Range $\Gamma, \Lambda \in \mathcal{H}_{n}$ and consider

$$
\begin{aligned}
\left\langle Q_{\Lambda}, \Sigma\right\rangle & =\operatorname{tr}\left(Q_{\Lambda} \Sigma\right)=\operatorname{tr}\left[\left(\Pi_{B}^{\perp} \Lambda \Pi_{B}^{\perp}-A^{*} \Pi_{B}^{\perp} \Lambda \Pi_{B}^{\perp} A\right) \Sigma\right] \\
& =\operatorname{tr}\left[\left(\Pi_{B}^{\perp} \Sigma \Pi_{B}^{\perp}-\Pi_{B}^{\perp} A \Sigma A^{*} \Pi_{B}^{\perp}\right) \Lambda\right] \\
& =\operatorname{tr}\left[\Pi_{B}^{\perp}\left(\Sigma-A \Sigma A^{*}\right) \Pi_{B}^{\perp} \Lambda\right]=0
\end{aligned}
$$

where we employed condition (7).

Thus, the original Problem 1 is reduced to finding $\hat{\Lambda}$ satisfying (25) and such that $\Sigma_{\hat{\wedge}}$ satisfies (7). This is accomplished in the next section via duality theory.

\section{DUAL PRoblem}

The dual functional takes form

$$
\begin{aligned}
\tilde{J}(\Lambda)= & \frac{1}{2} \log \operatorname{det} \Sigma_{\Lambda}^{-1}+\frac{1}{2} \operatorname{tr}\left(\hat{\Sigma}^{-1} \Sigma_{\Lambda}\right) \\
& +\operatorname{tr}\left[\Lambda \Pi_{B}^{\perp}\left(\Sigma_{\Lambda}-A \Sigma_{\Lambda} A^{*}\right) \Pi_{B}^{\perp}\right] \\
= & \frac{1}{2} \operatorname{tr}\left[\log \Sigma_{\Lambda}^{-1}+\hat{\Sigma}^{-1} \Sigma_{\Lambda}+2 \Lambda \Pi_{B}^{\perp}\left(\Sigma_{\Lambda}-A \Sigma_{\Lambda} A^{*}\right) \Pi_{B}^{\perp}\right] \\
= & \frac{1}{2} \operatorname{tr}\left[\log \Sigma_{\Lambda}^{-1}+\left(\hat{\Sigma}^{-1}+2 \Pi_{B}^{\perp} \Lambda \Pi_{B}^{\perp}-2 A^{*} \Pi_{B}^{\perp} \Lambda \Pi_{B}^{\perp} A\right) \Sigma_{\Lambda}\right] \\
= & \frac{1}{2} \operatorname{tr}\left[\log \Sigma_{\Lambda}^{-1}+\Sigma_{\Lambda}^{-1} \Sigma_{\Lambda}\right]=\frac{1}{2} \operatorname{tr}\left[\log \Sigma_{\Lambda}^{-1}+I\right] \\
= & \frac{1}{2} \operatorname{tr}\left[\log \left(\hat{\Sigma}^{-1}+2 Q_{\Lambda}\right)+I\right] .
\end{aligned}
$$

In order to find $\hat{\Lambda}$, we must maximize this function over the set

$$
\mathcal{L}:=\left\{\Lambda \in \mathcal{H}_{n} \mid \hat{\Sigma}^{-1}+2 Q_{\Lambda}>0\right\} .
$$

The dual problem is equivalent to minimize the following function over $\mathcal{L}$ :

$$
J(\Lambda):=-\frac{1}{2} \operatorname{tr} \log \left(\hat{\Sigma}^{-1}+2 Q_{\Lambda}\right) .
$$

To perform this minimization it is convenient to restrict our attention to a subset of $\mathcal{L}$ defined as follows. Consider the map

$$
\begin{aligned}
\varphi: \mathcal{H}_{n} & \rightarrow \mathcal{H}_{n} \\
\Lambda & \mapsto \Pi_{B}^{\perp} \Lambda \Pi_{B}^{\perp} .
\end{aligned}
$$

Such a map is self-adjoint because

$$
\langle\varphi(\Lambda), \Lambda\rangle=\operatorname{tr}\left(\Pi_{B}^{\perp} \Lambda \Pi_{B}^{\perp} \Lambda\right)=\operatorname{tr}\left(\Lambda \Pi_{B}^{\perp} \Lambda \Pi_{B}^{\perp}\right)=\langle\Lambda, \varphi(\Lambda)\rangle .
$$

Thus, $\operatorname{ker} \varphi=[\text { Range } \varphi]^{\perp}$. Suppose now that $J$ takes the minimum value in $\hat{\Lambda} \in \mathcal{L}$ and let $M \in[\text { Range } \varphi]^{\perp}$. It is easy to see that

$$
J(\hat{\Lambda}+M)=J(\hat{\Lambda})
$$

so that the search for the solution of the dual problem can be restricted to the set

$$
\mathcal{L}_{\varphi}:=\mathcal{L} \cap \text { Range } \varphi .
$$

Lemma 5.1: Consider $J: \mathcal{L} \rightarrow \mathbb{R}$. Then:

1) $J$ is strictly convex on $\mathcal{L}_{\varphi}$.

2) $J \in \mathcal{C}^{\infty}(\mathcal{L})$.

See Appendix A for the proof.

Corollary 5.1: The dual problem

$$
\text { Find } \Lambda \in \mathcal{L}_{\varphi} \text { minimizing } J(\Lambda)
$$

is a convex optimization problem which admits at most one solution.

We now tackle the existence issue for the dual problem. To this aim, we need a preliminary technical result whose proof is postponed to Appendix B.

Proposition 5.1: $\mathcal{L}_{\varphi}$ is an open and bounded set. 
We are now ready to prove existence of the minimum point.

Theorem 5.1: The dual functional (31) has a unique minimum point in $\mathcal{L}_{\varphi}$.

Proof: In view of Corollary 5.1, we only need to show that $J$ takes a minimum value on $\mathcal{L}_{\varphi}$. First we observe that $J$ is continuous on its domain. We now demonstrate that $J$ is inf-compact, i.e., the image of $(-\infty, r]$ under the map $J^{-1}$ is a compact set. It is then sufficient to apply Weierstrass theorem which states that a continuous function defined on a compact set admits a minimum. Indeed, observing that $J(0)=(1 / 2) \log \operatorname{det} \hat{\Sigma}$, we can restrict the search for a minimum point to the image of $(-\infty,(1 / 2) \log \operatorname{det} \hat{\Sigma}]$ under $J^{-1}$. Since, as stated in Proposition 5.1, $\mathcal{L}_{\varphi}$ is a bounded set, to prove inf-compactness of $J$ it is sufficient to show that

$$
\lim _{\Lambda \rightarrow \partial \mathcal{L}_{\varphi}} J(\Lambda)=+\infty .
$$

Notice that $\partial \mathcal{L}_{\varphi}$ is the set of $\Lambda \in$ Range $\varphi$ for which

$$
\hat{\Sigma}^{-1}+2 Q_{\Lambda}
$$

is a singular positive semidefinite matrix. Thus, for $\Lambda \rightarrow \partial \mathcal{L}_{\varphi}$ all the eigenvalues of (37) remain bounded and at least one of them tends to $0^{+}$. We denote with $\lambda_{1}, \ldots, \lambda_{n}>0$ the eigenvalues of $\Lambda$ and, without loss generality, we suppose that, for $\Lambda \rightarrow \partial \mathcal{L}_{\varphi}, \lambda_{1} \rightarrow 0^{+}$. Hence

$$
\begin{aligned}
\lim _{\Lambda \rightarrow \partial \mathcal{L}_{\varphi}} J(\Lambda) & =\lim _{\lambda_{1} \rightarrow 0^{+}}-\frac{1}{2} \log \prod_{i=1}^{n} \lambda_{i} \\
& =\lim _{\lambda_{1} \rightarrow 0^{+}} \sum_{i=1}^{n} \log \frac{1}{\sqrt{\lambda_{i}}}=+\infty .
\end{aligned}
$$

Corollary 5.2: The set

$$
\tilde{S}:=\left\{\Lambda \in \mathcal{L}_{\varphi} \mid J(\Lambda) \leq J(0)=\frac{1}{2} \log \operatorname{det} \hat{\Sigma}\right\}
$$

is compact.

\section{MAtricial Newton Algorithm}

In this section, we present a matricial Newton algorithm with backtracking stage for finding the minimum point of $J$ over $\mathcal{L}_{\varphi}$. To this end we introduce the linear functional

$$
\nabla J_{\Lambda}=\nabla J_{\Lambda}(\cdot):=\delta J(\Lambda, \cdot)
$$

which may be interpreted as the gradient of $J$ at $\Lambda$. Here, $\delta J(\Lambda, \delta \Lambda)$ is the first variation of $J$ at $\Lambda$ in direction $\delta \Lambda$. The bilinear form

$$
\mathcal{H}_{\Lambda}=\mathcal{H}_{\Lambda}(\cdot, \cdot):=\delta^{2} J(\Lambda, \cdot, \cdot)
$$

may be interpreted as the Hessian of $J$ at $\Lambda$. Here, $\delta^{2} J\left(\Lambda, \delta \Lambda_{1}, \delta \Lambda_{2}\right)$ is the second variation of $J$ at $\Lambda$ in directions $\delta \Lambda_{1}, \delta \Lambda_{2}$. The algorithm steps are the following:

1) Set the initial condition $\Lambda_{0}=0 \in \mathcal{L}_{\varphi}$.
2) At each iteration, compute the Newton step $\delta \Lambda_{i}$ over Range $\varphi$ by solving the following equation:

$$
\mathcal{H}_{\Lambda_{i}}\left(\delta \Lambda_{i}, \cdot\right)=-\nabla J_{\Lambda_{i}}(\cdot)
$$

where the gradient $\nabla J$ and the Hessian $\mathcal{H}$ are defined by (40) and (41), respectively. Taking into account (64) and (65) in the proof of Lemma 5.1 in Appendix A, the latter equation may be written explicitly as

$$
\begin{aligned}
2 \Pi_{B}^{\perp}\left[\Sigma_{\Lambda_{i}} Q_{\delta \Lambda_{i}} \Sigma_{\Lambda_{i}}-A \Sigma_{\Lambda_{i}} Q_{\delta \Lambda_{i}} \Sigma_{\Lambda_{i}} A^{*}\right] \Pi_{B}^{\perp} & = \\
& =-\Pi_{B}^{\perp}\left(A \Sigma_{\Lambda_{i}} A^{*}-\Sigma_{\Lambda_{i}}\right) \Pi_{B}^{\perp} .
\end{aligned}
$$

3) Set $t_{i}^{0}=1$, and let $t_{i}^{k+1}=t_{i}^{k} / 2$ until both of the following conditions hold:

$$
\begin{aligned}
\hat{\Sigma}^{-1}+2 Q_{\Lambda_{i}+t_{i}^{k} \delta \Lambda_{i}} & >0 \\
J\left(\Lambda_{i}+t_{i}^{k} \delta \Lambda_{i}\right) & <J\left(\Lambda_{i}\right)+\alpha t_{i}^{k} \operatorname{tr}\left[\nabla J_{\Lambda_{i}} \delta \Lambda_{i}\right]
\end{aligned}
$$

where $\alpha \in(0,1 / 2)$ is a real constant. Notice that, since $\Lambda_{i}+t_{i}^{k} \delta \Lambda_{i} \in$ Range $\varphi$, condition (44) implies that

$$
\Lambda_{i}+t_{i}^{k} \delta \Lambda_{i} \in \mathcal{L}_{\varphi} .
$$

4) Set $\Lambda_{i+1}=\Lambda_{i}+t_{i}^{k} \delta \Lambda_{i}$.

5) Repeat steps 2, 3 and 4 until the condition $\left\|\nabla J_{\Lambda_{i}}\right\|_{2}<\varepsilon$ is satisfied, where $\varepsilon$ is a (small) tolerance threshold, then set $\hat{\Lambda}=\Lambda_{i}$.

We suggest the following procedure to solve (42) by taking into account the explicit form (43):

1) Take a basis $\left\{\Omega_{1}, \ldots, \Omega_{l}\right\}$ of Range $\varphi$.

2) Compute

$$
Y=\Pi_{B}^{\perp}\left(\Sigma_{\Lambda_{i}}-A \Sigma_{\Lambda_{i}} A^{*}\right) \Pi_{B}^{\perp}
$$

3) For each $\Omega_{k}$, compute:

$$
Y_{k}=2 \Pi_{B}^{\perp}\left[\Sigma_{\Lambda_{i}} Q_{\Omega_{k}} \Sigma_{\Lambda_{i}}-A \Sigma_{\Lambda_{i}} Q_{\Omega_{k}} \Sigma_{\Lambda_{i}} A^{*}\right] \Pi_{B}^{\perp} .
$$

4) Solve, by means of linear algebraic methods (the MoorePenrose pseudoinverse), the equation

$$
\sum_{k} \alpha_{k} Y_{k}=Y .
$$

5) By linearity, the solution to (42) is

$$
\delta \Lambda_{i}=\sum_{k} \alpha_{k} \Omega_{k} \in \text { Range } \varphi .
$$

This algorithm converges globally: In the first stage, it converges in linear way. In the last stage, in quadratic way. The proof of these facts is postponed to Appendix C.

\section{PERformance COMPARISON}

In this section, we use the following notation:

- PJ method to denote the projection method outlined in the last part of Section III;

- ME method to denote the maximum entropy method introduced in Section IV. 


\section{A. Performance Comparison Procedure}

Suppose that we have a finite sequence $y_{1}, \ldots, y_{N}$ extracted from a sample path of a zero-mean, weakly stationary discretetime process $\left\{y_{t}\right\}_{t=-\infty}^{+\infty}$. We want to compare the estimates $\Sigma_{P J}$ and $\Sigma_{M E}$ obtained by the PJ and ME methods, respectively. In order to make the comparison reasonably independent of the specific data set, we average over $M=500$ experiments performed with sequences extracted from different sample paths. We are now ready to describe the comparison procedure:

- Fix the transfer function $G(z)$.

- At the $k$-th experiment $G(z)$ is fed by the data $\left\{y_{i}^{k}\right\}_{i=1}^{N}$ and we collect the output data $\left\{x_{i}^{k}\right\}_{i=1}^{N}$.

- Compute the consistent estimate $\hat{\Sigma}(k)$ of the covariance matrix of the output from $\left\{x_{i}^{k}\right\}_{i=\tilde{N}}^{N}$, with $\tilde{N}<N$, as in (15). Note that the first $\tilde{N}-1$ output samples $x_{1}^{k}, \ldots, x_{\tilde{N}-1}^{k}$ are discarded so that the filter can be considered to operate in steady state.

- From $\hat{\Sigma}(k)$, estimate $\Sigma_{P J}(k)$ and $\Sigma_{M E}(k)$ using PJ and ME method respectively.

- Compute the relative error norm ${ }^{1}$ between $\Sigma$ and its estimates $\Sigma_{P J}(k)$ and $\Sigma_{M E}(k)$

$$
e_{P J}(k)=\frac{\left\|\Sigma_{P J}(k)-\Sigma\right\|}{\|\Sigma\|}, e_{M E}(k)=\frac{\left\|\Sigma_{M E}(k)-\Sigma\right\|}{\|\Sigma\|} .
$$

- When the experiments are completed, compute the mean and the variance of the relative error norm

$$
\begin{aligned}
\mu_{P J} & =\frac{1}{M} \sum_{k=1}^{M} e_{P J}(k), \mu_{M E}=\frac{1}{M} \sum_{k=1}^{M} e_{M E}(k), \\
\sigma_{P J}^{2} & =\frac{1}{M} \sum_{k=1}^{M}\left(e_{P J}(k)-\mu_{P J}\right)^{2}, \\
\sigma_{M E}^{2} & =\frac{1}{M} \sum_{k=1}^{M}\left(e_{M E}(k)-\mu_{M E}\right)^{2} .
\end{aligned}
$$

- Count the times that the $P J$ method adjusts the projected estimation $\hat{\Sigma}_{\Gamma}(k)$ by adding to it the quantity $\varepsilon \Sigma_{+}$. This number is denoted as $\sharp F$.

The output of this procedure are the parameters $\mu_{j}, \sigma_{j}^{2}$ and $\sharp F$. Clearly, the smaller these parameters, the better estimation is expected.

\section{B. Simulation Results for the Real Scalar Case}

We choose a real scalar process $y$ with a high-order spectral density $\Phi$ (represented by the solid line in Fig. 1). The bank of filters has the following structure

$$
A=\left[\begin{array}{cccccc}
a_{1} & 1 & 0 & 0 & 0 & 0 \\
0 & a_{2} & 1 & 0 & 0 & 0 \\
0 & 0 & a_{3} & 1 & 0 & 0 \\
0 & 0 & 0 & a_{4} & 1 & 0 \\
0 & 0 & 0 & 0 & a_{5} & 1 \\
0 & 0 & 0 & 0 & 0 & a_{6}
\end{array}\right], B=\left[\begin{array}{l}
0 \\
1 \\
0 \\
1 \\
0 \\
1
\end{array}\right]
$$

First we choose

$$
a_{1}=a_{2}=a_{3}=0.4, \quad a_{4}=a_{5}=a_{6}=0.5 .
$$

${ }^{1}$ Here the norm $\|\cdot\|$ is the spectral norm. i.e., the matrix norm induced by the Euclidean norm in $\mathbb{C}^{n}$.

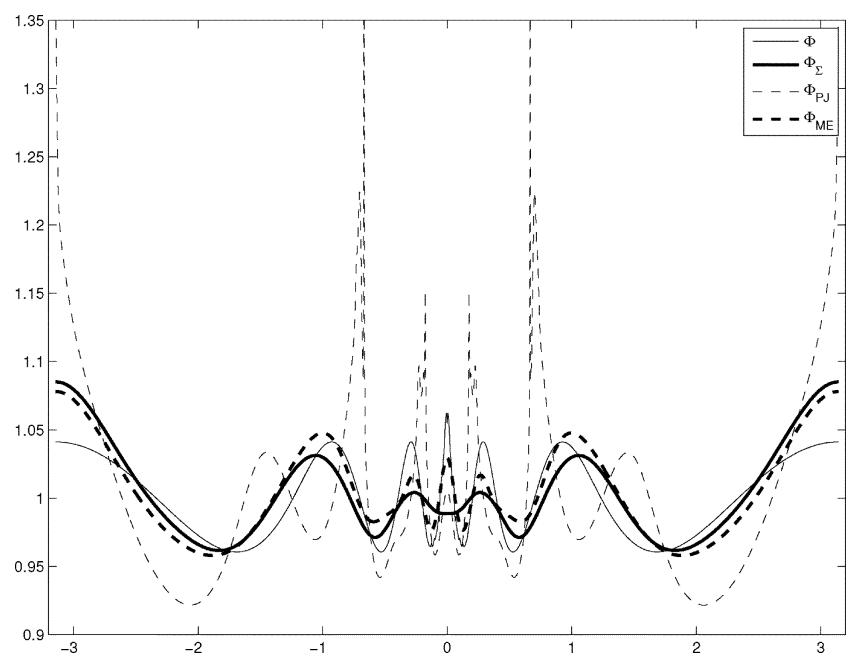

Fig. 1. Mean spectra comparison using Prior-THREE spectral estimation method, with the bank of filters (52)-(53).

TABLE I

PARAMETERS $\mu_{P J}, \mu_{M E}, \sigma_{P J}^{2}, \sigma_{M E}^{2}, \sharp F$ FOR $A, B$ GIVEN BY (52)-(53)

\begin{tabular}{|c|c|c|c|c|c|}
\hline$N$ & $\mu_{P J}$ & $\mu_{M E}$ & $\sigma_{P J}^{2}$ & $\sigma_{M E}^{2}$ & $\sharp F$ \\
\hline 300 & 0.19827 & 0.19045 & 0.026206 & 0.018779 & 11 \\
\hline 500 & 0.14329 & 0.14169 & 0.013137 & 0.011047 & 1 \\
\hline 700 & 0.12701 & 0.1269 & 0.0092071 & 0.0091365 & 0 \\
\hline 1000 & 0.10679 & 0.10677 & 0.0064781 & 0.0064709 & 0 \\
\hline
\end{tabular}

In this case, the true covariance $\Sigma$ of the process $\left\{x_{i}\right\}_{i=-\infty}^{+\infty}$ has the following eigenvalues $\lambda_{1}=0.2408, \lambda_{2}=0.4775$, $\lambda_{3}=1.9235, \lambda_{4}=2.8125, \lambda_{5}=21.1455, \lambda_{6}=285.2539$. Thus $\Sigma$ has a condition number of the order of $10^{3}$. In Table I, we present the results obtained for different lengths $N$ of the observed sequences $\left\{y_{i}^{k}\right\}_{i=1}^{N}$. In this case, the method developed in this paper appears produce only a very marginal improvement with respect to the projection method. Moreover, as $N$ increases, $\mu$ and $\sigma^{2}$ decrease for both methods: In fact, $\hat{\Sigma} \rightarrow \Sigma$ with probability one as $N \rightarrow+\infty$. Therefore, as $N$ increases, the performances of the two methods are more and more similar. This picture, however, changes dramatically if the time-constants of the dynamics of the filter $G(z)$ are significatively different. Consider, for example, a filters bank with the same structure (52) but with

$$
a_{1}=0.3, a_{2}=0.4, a_{3}=0.5, a_{4}=0.6, a_{5}=0.7, a_{6}=0.8 .
$$

In this case, the eigenvalues of $\Sigma$ are $\lambda_{1}=0.3, \lambda_{2}=0.7$, $\lambda_{3}=2.1, \lambda_{4}=8.5, \lambda_{5}=123.8, \lambda_{6}=3862.3$. Thus, the condition number of $\Sigma$ is of the order of $10^{4}$. In Table II we present the results obtained for different lengths $N$ of the observed sequences $\left\{y_{i}^{k}\right\}_{i=1}^{N}$.

In this situation, the condition number of $\Sigma$ is larger than in the previous case. Thus, the projection of $\hat{\Sigma}$ (that is a perturbed version of $\Sigma$ ) onto Range $\Gamma$ yields a matrix $\hat{\Sigma}_{\Gamma}$ that, in many cases, fails to be positive definite (or even positive semidefinite). This explains why the number of failures $\sharp F$ is significant. Recall that, when the projection fails to be positive definite, the PJ method adjusts $\hat{\Sigma}_{\Gamma}$ by adding a positive definite matrix $\Sigma_{+}$ belonging to Range $\Gamma$. For each experiment, $\Sigma_{+}$is the same. 
TABLE II

PARAMETERS $\mu_{P J}, \mu_{M E}, \sigma_{P J}^{2}, \sigma_{M E}^{2}, \sharp F$ FOR $A, B$ GIVEN BY (52)-(54)

\begin{tabular}{|c|c|c|c|c|c|}
\hline$N$ & $\mu_{P J}$ & $\mu_{M E}$ & $\sigma_{P J}^{2}$ & $\sigma_{M E}^{2}$ & $\sharp F$ \\
\hline 300 & 1.6509 & 0.24068 & 8.1929 & 0.030232 & 197 \\
\hline 500 & 0.99964 & 0.17711 & 3.1593 & 0.018377 & 141 \\
\hline 700 & 0.60446 & 0.15266 & 1.2813 & 0.01464 & 99 \\
\hline 1000 & 0.49333 & 0.13648 & 0.95235 & 0.011395 & 78 \\
\hline
\end{tabular}

TABLE III

PARAMETERS $\mu_{P J}, \mu_{M E}, \sigma_{P J}^{2}, \sigma_{M E}^{2}, \sharp F$ FOR $A, B$ GIVEN BY (55)-(56)

\begin{tabular}{|c|c|c|c|c|c|}
\hline$N$ & $\mu_{P J}$ & $\mu_{M E}$ & $\sigma_{P J}^{2}$ & $\sigma_{M E}^{2}$ & $\sharp F$ \\
\hline 300 & 0.3372 & 0.17694 & 0.46351 & 0.017164 & 33 \\
\hline 500 & 0.16107 & 0.1431 & 0.043056 & 0.011703 & 6 \\
\hline 700 & 0.12044 & 0.11778 & 0.010054 & 0.006674 & 1 \\
\hline 1000 & 0.09712 & 0.09696 & 0.005345 & 0.005333 & 0 \\
\hline
\end{tabular}

Hence, the adjustment cannot provide a good estimate of $\Sigma$. This is the heuristic reason why, in this case, the estimates provided by our method largely outperform those obtained by the projection method. Indeed, even increasing $N$ to 1000 (so that the observed sequences are pretty long), the differences in the performances remain remarkable.

Remark 7.1: We hasten to anticipate that even in the case of the filters bank (52)-(53), with $N=500$ or larger, when the estimation errors of the PJ and ME methods have practically the same mean and variance, the THREE-like spectral estimator performs much better when initialized with $\Sigma_{M E}$ than when initialized with $\Sigma_{P J}$ (see next section).

\section{Simulation Results for the Real Multivariable Case}

We consider a bivariate real process $y$ with a high-order spectral density $\Phi$. As for the scalar case, we consider two filters banks with the same structure

$$
A=\left[\begin{array}{cccccc}
a_{1} & 1 & 0 & 0 & 0 & 0 \\
0 & a_{1} & 1 & 0 & 0 & 0 \\
0 & 0 & a_{1} & 0 & 0 & 0 \\
0 & 0 & 0 & a_{2} & 1 & 0 \\
0 & 0 & 0 & 0 & a_{2} & 1 \\
0 & 0 & 0 & 0 & 0 & a_{2}
\end{array}\right], B=\left[\begin{array}{ll}
0 & 0 \\
1 & 0 \\
0 & 1 \\
0 & 0 \\
1 & 0 \\
0 & 1
\end{array}\right] .
$$

In the first case

$$
a_{1}=0.55, a_{2}=0.65 \text {. }
$$

In this case, the true $\Sigma$ has the following eigenvalues: $\lambda_{1}=$ 0.0031, $\lambda_{2}=0.0120, \lambda_{3}=0.9863, \lambda_{4}=2.3744, \lambda_{5}=$ $8.1872, \lambda_{6}=84.0289$. The corresponding error means and variances for the two estimation methods PJ and ME are reported in Table III for different values of the length $N$ of the observed data sequences $\left\{y_{i}^{k}\right\}_{i=1}^{N}$.

The second filters bank has the same structure (55), but the eigenvalues of $A$ are closer to the unit circle

$$
a_{1}=0.6, a_{2}=0.7 \text {. }
$$

In this case, the true $\Sigma$ has the following eigenvalues: $\lambda_{1}=$ 0.0034, $\lambda_{2}=0.0169, \lambda_{3}=1.4706, \lambda_{4}=2.9195, \lambda_{5}=$ 11.8157, $\lambda_{6}=159.1730$. The corresponding error means and
TABLE IV

PARAMETERS $\mu_{P J}, \mu_{M E}, \sigma_{P J}^{2}, \sigma_{M E}^{2}, \sharp F$ FOR $A, B$ GIVEN BY (55)-(57)

\begin{tabular}{|c|c|c|c|c|c|}
\hline$N$ & $\mu_{P J}$ & $\mu_{M E}$ & $\sigma_{P J}^{2}$ & $\sigma_{M E}^{2}$ & $\sharp F$ \\
\hline 300 & 1.0234 & 0.20392 & 3.5055 & 0.022113 & 93 \\
\hline 500 & 0.45285 & 0.14658 & 1.4239 & 0.013541 & 35 \\
\hline 700 & 0.25175 & 0.12041 & 0.55549 & 0.0082425 & 16 \\
\hline 1000 & 0.16576 & 0.11102 & 0.22098 & 0.006274 & 7 \\
\hline
\end{tabular}

TABLE V

PARAMETERS $\mu_{P J}, \mu_{M E}, \sigma_{P J}^{2}, \sigma_{M E}^{2}, \sharp F$ FOR $A, B$ GIVEN By (58)

\begin{tabular}{|c|c|c|c|c|c|}
\hline$N$ & $\mu_{P J}$ & $\mu_{M E}$ & $\sigma_{P J}^{2}$ & $\sigma_{M E}^{2}$ & $\sharp F$ \\
\hline 300 & 0.6861 & 0.1481 & 0.79881 & 0.011669 & 148 \\
\hline 500 & 0.32225 & 0.11871 & 0.3374 & 0.006533 & 59 \\
\hline 700 & 0.20526 & 0.10044 & 0.18662 & 0.004495 & 29 \\
\hline 1000 & 0.13541 & 0.08525 & 0.0919 & 0.003015 & 14 \\
\hline
\end{tabular}

variances are reported in the Table IV. As it can be observed from the tables, the scenario is the same as in the scalar case: The ME method performs remarkably better than the PJ method, particularly for the second filters bank.

\section{Simulation Results for the Complex Case}

So far we have considered only real examples because this situation is more common in control engineering applications. Since the theory has, however, been developed for the more general complex case, we also include the following complex example where the process $y$ is a high order (the McMillan degree of the corresponding spectral density $\Phi$ is 80) complex-valued scalar process. Let $A$ and $B$ be defined by

$$
A=\left[\begin{array}{cccccc}
a_{1} & 1 & 0 & 0 & 0 & 0 \\
0 & a_{2} & 1 & 0 & 0 & 0 \\
0 & 0 & a_{3} & 1 & 0 & 0 \\
0 & 0 & 0 & a_{4} & 1 & 0 \\
0 & 0 & 0 & 0 & a_{5} & 1 \\
0 & 0 & 0 & 0 & 0 & a_{6}
\end{array}\right], \quad B=\left[\begin{array}{l}
0 \\
0 \\
0 \\
0 \\
0 \\
1
\end{array}\right]
$$

where $a_{i}=0.7 \mathrm{e}^{\mathrm{j} \omega_{i}}$ and $\omega_{1}:=0.8148, \omega_{2}:=0.9058, \omega_{3}:=$ $0.1270, \omega_{4}:=0.9133, \omega_{5}:=0.6324, \omega_{6}:=0.0976$. The eigenvalues of the matrix $\Sigma$ are $\lambda_{1}=0.5, \lambda_{2}=3.06, \lambda_{3}=$ $17.65, \lambda_{4}=132.79, \lambda_{5}=1.55 \cdot 10^{3}, \lambda_{6}=2.67 \cdot 10^{4}$. Table V, where the performances of our method are compared to those of the projection method, shows that also in this case our approach is particularly convenient.

\section{APPLiCATION TO SPECTRAL ESTIMATION}

As mentioned in the introduction, there is a family of spectral estimation methods which need to be initialized with a positive definite estimate $\Sigma_{\circ} \in$ Range $\Gamma$ of the actual state covariance $\Sigma$. For example, in the case when the process $y$ is scalar, the THREE estimator [4] finds the maximum entropy spectrum $\hat{\Phi}$ satisfying the constraint

$$
\int G \hat{\Phi} G^{*}=\Sigma_{\circ}
$$

The estimated spectral density can be expressed in closed form (see [20]) as

$$
\hat{\Phi}=\left[G^{*} \Sigma_{\circ}^{-1} B\left(B^{*} \Sigma_{\circ}^{-1} B\right)^{-1} B^{*} \Sigma_{\circ}^{-1} G\right]^{-1} .
$$


In [10], this setting was generalized by introducing the possibility of considering prior information encoded in an a priori spectral density $\Psi$. More precisely, a Kullback-Leibler type of pseudo-distance for coercive spectra was introduced

$$
\mathbb{D}(\Psi \| \Phi)=\int \Psi \log \left(\frac{\Psi}{\Phi}\right)
$$

and the corresponding constrained approximation problem was solved. In the following, we will denote the corresponding estimator, in which the estimate is obtained by minimizing (61) under constraint (59), as "Prior-THREE." We observe that (60) provides, also in the multivariable case, the maximum entropy spectral density satisfying (59), cf. [20]. The generalization to the multivariable case of the Prior-THREE algorithm, however, is more challenging since the variational analysis cannot be carried through. In [31] and [32], this generalization has been successfully carried out in a different metric induced by a Hellingertype distance.

Next, we compare the estimated spectral densities, obtained by one of the THREE-like spectral estimation algorithms, when initialized with the true variance $\Sigma$ and with the two estimates $\Sigma_{P J}$ and $\Sigma_{M E}$. We stress that, while the results of Section VII compare the estimated covariance $\Sigma_{M E}$ or $\Sigma_{P J}$ to the true $\Sigma$, the following comparison evaluates the different performances directly in terms of the main applications of the methods, i.e., spectral estimation and spectrum approximation.

\section{A. Simulation Results for the Scalar Case Using the Prior-Three Algorithm}

From the procedure presented in Section VII-A, we get the state covariance estimates $\Sigma_{P J}(k)$ and $\Sigma_{M E}(k)$ for $k=1 \ldots, M$. Thus, we exploit this set of estimates (with $M=500$ experiments and $N=500$ ) as input state covariances for the chosen spectral estimation method. Here we describe the case of the method Prior-THREE:

- We consider a prior spectral density $\Psi_{k}\left(\mathrm{e}^{\mathrm{j} \vartheta}\right)$ that may depend on the data $\left\{y_{i}^{k}\right\}_{i=1}^{N}$ and hence is indexed on $k$.

- For each experiment $k$, we compute the spectrum estimate $\hat{\Phi}_{T, k}\left(\mathrm{e}^{\mathrm{j} \vartheta}\right)$ using Prior-THREE with inputs $\Psi_{k}\left(\mathrm{e}^{\mathrm{j} \vartheta}\right)$ and the true variance $\Sigma$.

- For each experiment $k$, we compute the spectrum estimates $\hat{\Phi}_{P J, k}\left(\mathrm{e}^{\mathrm{j} \vartheta}\right)$, and $\hat{\Phi}_{M E, k}\left(\mathrm{e}^{\mathrm{j} \vartheta}\right)$ of the Prior-THREE algorithm using the same "a priori" spectral density $\Psi_{k}\left(\mathrm{e}^{\mathrm{j} \vartheta}\right)$ and taking $\Sigma_{P J}(k)$ and $\Sigma_{M E}(k)$, respectively, as state variance.

- When the spectral estimates are completed, we compute the mean estimates

$$
\begin{aligned}
\Phi_{T}\left(\mathrm{e}^{\mathrm{j} \vartheta}\right) & :=\frac{1}{M} \sum_{k=1}^{M} \hat{\Phi}_{T, k}\left(\mathrm{e}^{\mathrm{j} \vartheta}\right) \\
\Phi_{P J}\left(\mathrm{e}^{\mathrm{j} \vartheta}\right) & :=\frac{1}{M} \sum_{k=1}^{M} \hat{\Phi}_{P J, k}\left(\mathrm{e}^{\mathrm{j} \vartheta}\right) \\
\Phi_{M E}\left(\mathrm{e}^{\mathrm{j} \vartheta}\right) & :=\frac{1}{M} \sum_{k=1}^{M} \hat{\Phi}_{M E, k}\left(\mathrm{e}^{\mathrm{j} \vartheta}\right)
\end{aligned}
$$

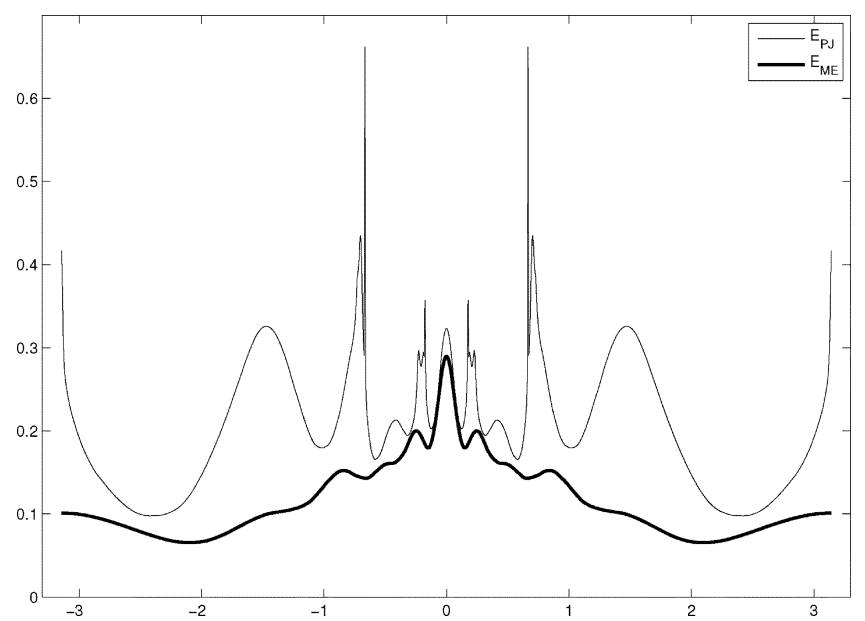

Fig. 2. Mean error norm comparison using Prior-THREE spectral estimation method, with the bank of filters (52)-(53).

and the mean of the error norm for each method with respect to $\hat{\Phi}_{T, k}\left(\mathrm{e}^{\mathrm{j} \vartheta}\right)$

$$
\begin{aligned}
E_{P J}(\vartheta) & :=\frac{1}{M} \sum_{k=1}^{M}\left|\hat{\Phi}_{P J, k}\left(\mathrm{e}^{\mathrm{j} \vartheta}\right)-\hat{\Phi}_{T, k}\left(\mathrm{e}^{\mathrm{j} \vartheta}\right)\right| \\
E_{M E}(\vartheta) & :=\frac{1}{M} \sum_{k=1}^{M}\left|\hat{\Phi}_{M E, k}\left(\mathrm{e}^{\mathrm{j} \vartheta}\right)-\hat{\Phi}_{T, k}\left(\mathrm{e}^{\mathrm{j} \vartheta}\right)\right| .
\end{aligned}
$$

Remark 8.1: Notice that the very same procedure may be employed to deal with the THREE estimation procedure which is just the special case of the Prior-THREE corresponding to the choice $\Psi_{k}\left(\mathrm{e}^{\mathrm{j} \vartheta}\right) \equiv 1$ for the prior spectral density.

Notice also that, in the above procedure, an essential degree of freedom is the filter bank $G(z)$. Indeed, the choice of $G(z)$ has profound implications (see [4], [10], [33] and [13]). In fact, it turns out that the spectrum estimate has better resolution in those sectors of the unit circle where more eigenvalues are located close to the unit circle.

To perform the comparison, we have chosen the two filters $G(z)$ used in Section VII-B and we have set the prior spectral density to be $\Psi_{k}(z):=W_{k}(z) W_{k}^{*}(z)$ where $W_{k}(z)=$ $\left[\hat{\sigma}_{e} c(z) / a(z)\right]_{k}$ is a three-order AR model estimated from the sequence $y_{1}^{k}, \ldots, y_{N}^{k}$ extracted from the $k$-th sample path of the process $y$.

In Fig. 1 the mean spectra corresponding to the filters bank (52)-(53) are depicted. In Fig. 2 the corresponding mean error norms are represented.

It is apparent that our method produces an estimate $\Sigma_{M E}$ for which the corresponding spectral density $\Phi_{M E}$ approximates the true $\Phi$ almost as well as the estimation produced starting from the true $\Sigma$, while the estimation corresponding to $\Sigma_{P J}$ is highly unsatisfactory. Notice also that, although in this case $\Sigma_{P J}$ and $\Sigma_{M E}$ appear quite similar (see the table in the previous section), the estimated spectra are very different and the ME method provides a considerable improvement, cf. Remark 7.1.

Figs. 3 and 4 show the mean spectra and the mean error norm, respectively, when the filters bank (52)-(54) is employed. As expected, in this case the inferior performance of the $P J$ method 


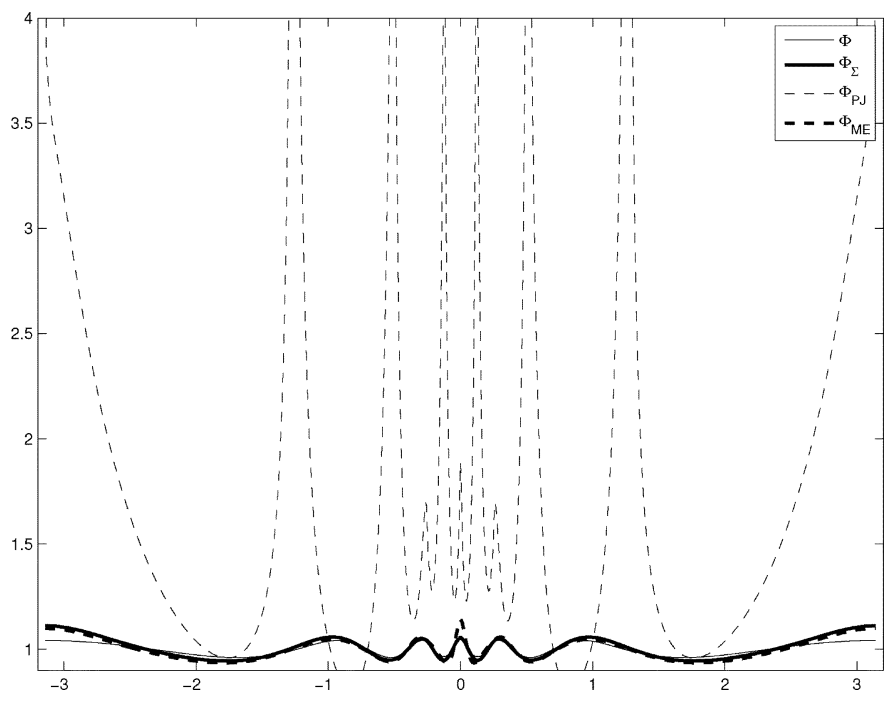

Fig. 3. Mean spectra comparison using Prior-THREE spectral estimation method, with the bank of filters (52)-(54).

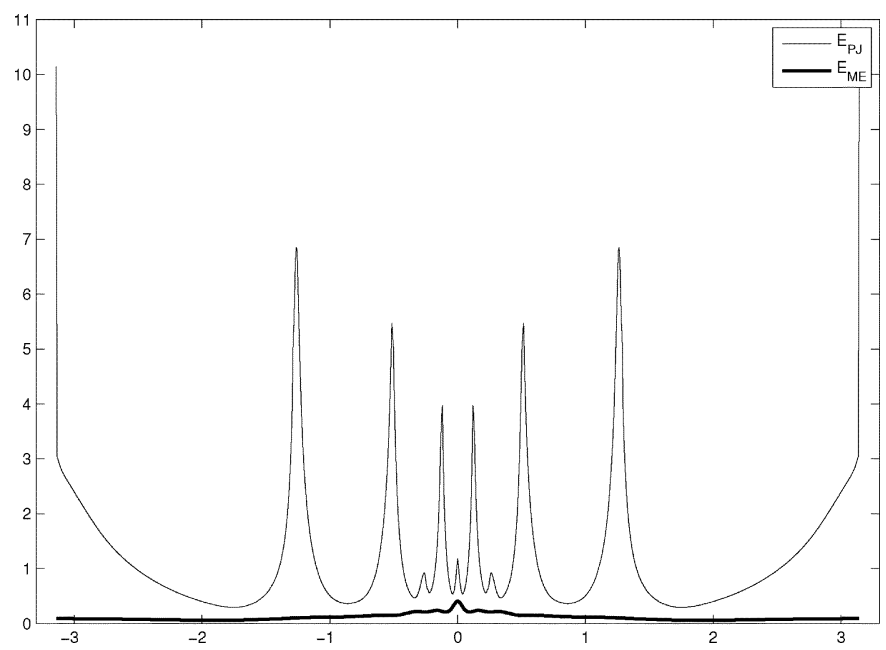

Fig. 4. Mean error norm comparison using Prior-THREE spectral estimation method, with the bank of filters (52)-(54).

when compared to the $M E$ method is more salient while the $M E$ method practically performs as well as the estimation $\Phi_{\Sigma}$ produced by employing the true $\Sigma$. Similar results are obtained when using the THREE estimator $\left(\Psi_{k}\left(\mathrm{e}^{\mathrm{j} \vartheta}\right) \equiv 1\right)$.

\section{B. Simulation Results for the Multivariable Case}

We have carried out this comparison along the very same lines of Section VIII-A employing the same $\Phi(z)$ and the same two filters $G(z)$ used in Section VII-C. The only differences with respect to Section VIII-A are the following:

1) For the spectral estimation, we have employed the maximum entropy estimator (60) in which we have plugged the true variance $\Sigma$ and the two estimates $\Sigma_{P J}$ and $\Sigma_{M E}$.

2) We have modified (63) by using the matrix induced norms in place of the absolute values.

3) We have illustrated only the mean of the errors norm since comparing the $2 \times 2$ spectral densities would require four pictures for each case.

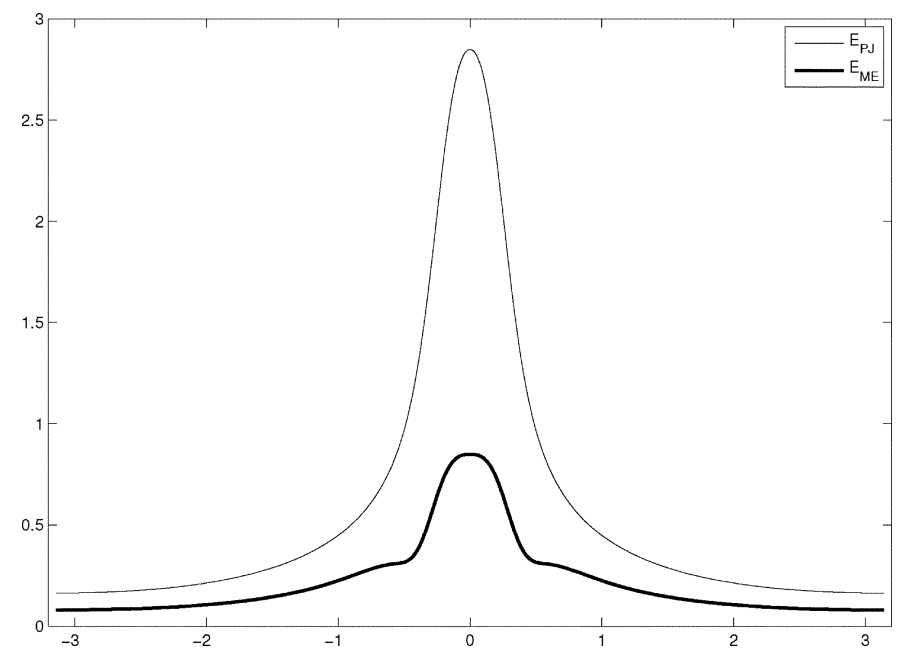

Fig. 5. Mean of the error norm comparison using maximum entropy spectral estimation method, with the bank of filters (55)-(56).

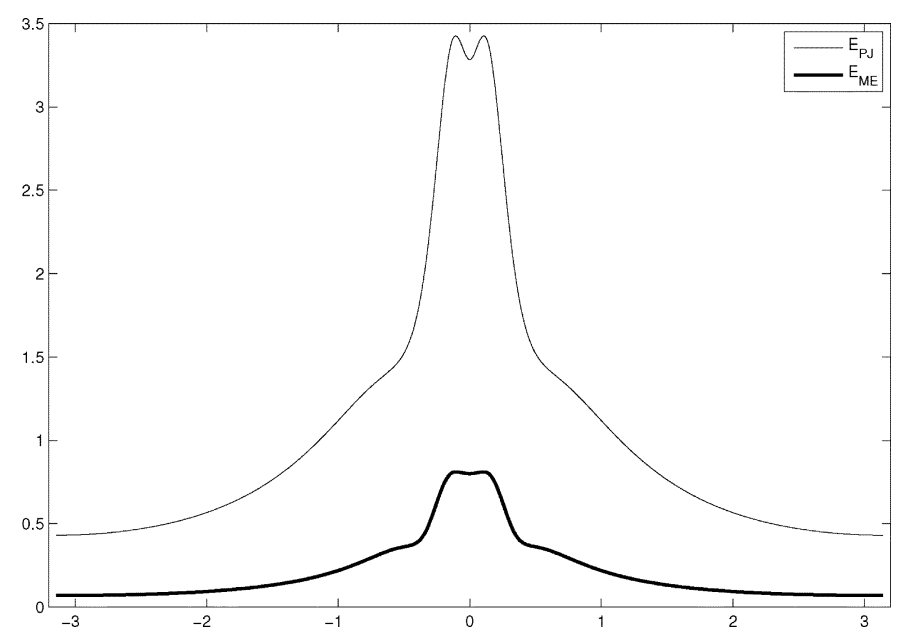

Fig. 6. Mean of the error norm comparison using maximum entropy spectral estimation method, with the bank of filters (55)-(57).

Fig. 5 shows the mean of the error norm in the case of filters bank (55)-(56). Although $\Sigma_{P J}$ and $\Sigma_{M E}$ are quite similar in this case (see the table in the previous section), the difference among the mean error norms is more evident and the ME method provides an estimate closer to the estimate obtained using the "true" $\Sigma$. Finally, in Fig. 6, the mean of the error norm is depicted for the case of the filters bank (55)-(57). The spectral estimate obtained using $\Sigma_{P J}$ is clearly unsatisfactory with respect to the one obtained using $\Sigma_{M E}$. In conclusion, the significant improvement in spectral estimation brought about by our method occurs also in the multivariable setting.

\section{CONCLUSION}

In this paper, we have proposed a new systematic approach to initializing the THREE-like spectral estimation algorithms. Our approach hinges on an ancillary maximum entropy problem whose theoretical and computational aspects have been here thoroughly investigated. It appears that, in several critical cases, the projection method of Section III provides a poor estimate of the covariance matrix $\Sigma$, compromising the quality of the spectral estimator. Moreover, simulation shows that, even when 
the projection-based estimate $\Sigma_{P J}$ looks close to our estimate $\Sigma_{M E}$, the spectral estimator initialized with $\Sigma_{M E}$ significantly outperforms the other one. Indeed, it often performs nearly as well as the estimator initialized with the true state covariance $\Sigma$.

\section{APPENDIX}

\section{A. Proof of Lemma 5.1:}

1) First of all, observe that $\mathcal{L}$ is an open, convex subset of $\mathcal{H}_{n}$. Moreover, since $J$ is the negative of a dual functional, it is convex. For $\delta \Lambda_{1} \in \mathcal{H}_{n}$, we compute the directional derivative

$$
\begin{aligned}
\delta J\left(\Lambda, \delta \Lambda_{1}\right) & =-\operatorname{tr}\left[\Sigma_{\Lambda}\left(\Pi_{B}^{\perp} \delta \Lambda_{1} \Pi_{B}^{\perp}-A^{*} \Pi_{B}^{\perp} \delta \Lambda_{1} \Pi_{B}^{\perp} A\right)\right] \\
& =-\operatorname{tr}\left[\Sigma_{\Lambda} Q \delta \Lambda_{1}\right] .
\end{aligned}
$$

The second variation, in directions $\delta \Lambda_{1}, \delta \Lambda_{2} \in \mathcal{H}_{n}$, is given by

$$
\begin{aligned}
\delta^{2} J\left(\Lambda, \delta \Lambda_{1}, \delta \Lambda_{2}\right)= & 2 \operatorname{tr}\left[\Sigma_{\Lambda}\left(\Pi_{B}^{\perp} \delta \Lambda_{2} \Pi_{B}^{\perp}-A^{*} \Pi_{B}^{\perp} \delta \Lambda_{2} \Pi_{B}^{\perp} A\right)\right. \\
& \left.\times \Sigma_{\Lambda}\left(\Pi_{B}^{\perp} \delta \Lambda_{1} \Pi_{B}^{\perp}-A^{*} \Pi_{B}^{\perp} \delta \Lambda_{1} \Pi_{B}^{\perp} A\right)\right] \\
= & 2 \operatorname{tr}\left[\Sigma_{\Lambda} Q_{\delta \Lambda_{2}} \Sigma_{\Lambda} Q_{\delta \Lambda_{1}}\right] .
\end{aligned}
$$

Consider now

$$
\begin{aligned}
\delta^{2} J(\Lambda, \delta \Lambda, \delta \Lambda) & =2 \operatorname{tr}\left[\Sigma_{\Lambda} Q_{\delta \Lambda} \Sigma_{\Lambda} Q_{\delta \Lambda}\right] \\
& =2 \operatorname{tr}\left[\Sigma_{\Lambda}^{1 / 2} Q_{\delta \Lambda} \Sigma_{\Lambda} Q_{\delta \Lambda} \Sigma_{\Lambda}^{1 / 2}\right]
\end{aligned}
$$

which, as expected, is a nonnegative quantity since $\Sigma_{\Lambda}$ is positive definite. Suppose now that $\delta \Lambda \in \mathcal{L}_{\varphi}$. The equation

$$
\Pi_{B}^{\perp} \delta \Lambda \Pi_{B}^{\perp}=A^{*} \Pi_{B}^{\perp} \delta \Lambda \Pi_{B}^{\perp} A+Q_{\delta \Lambda}
$$

is the Lyapunov equation associated to the $\left(A^{*}, Q_{\delta_{\Lambda}}\right)$ pair where we regard as the unknown $\Pi_{B}^{\perp} \delta \Lambda \Pi_{B}^{\perp}$. It follows that $\Pi_{B}^{\perp} \delta \Lambda \Pi_{B}^{\perp}$ can be expressed as

$$
\Pi_{B}^{\perp} \delta \Lambda \Pi_{B}^{\perp}=\sum_{t=0}^{\infty}\left(A^{*}\right)^{t} Q_{\delta \Lambda} A^{t} .
$$

Since $\delta \Lambda \in \mathcal{L}_{\varphi} \subset \operatorname{ker} \varphi^{\perp}$, from $\Pi_{B}^{\perp} \delta \Lambda \Pi_{B}^{\perp}=0$ it follows that $\delta \Lambda=0$. Thus, taking (68) into account, we have that $Q_{\delta \Lambda}=0$ implies $\delta \Lambda=0$. Accordingly, we have that $\delta^{2} J(\Lambda, \delta \Lambda, \delta \Lambda)$ is strictly positive for any non zero $\delta \Lambda \in \mathcal{L}_{\varphi}$, and consequently, $J$ is strictly convex on $\mathcal{L}_{\varphi}$.

2) Notice that the first and the second variation of $J$ exist and are continuous on $\mathcal{L}$. The same applies to the third variation in directions $\delta \Lambda_{1}, \delta \Lambda_{2}, \delta \Lambda_{3} \in \mathcal{H}_{n}$

$$
\begin{aligned}
& \delta^{3}\left(\Lambda, \delta \Lambda_{1}, \delta \Lambda_{2}, \delta \Lambda_{3}\right) \\
& =\delta\left\{2 \operatorname{tr}\left[\Sigma_{\Lambda} Q_{\delta \Lambda_{2}} \Sigma_{\Lambda} Q_{\delta \Lambda_{1}}\right], \delta \Lambda_{3}\right\} \\
& =2 \operatorname{tr}\left\{\delta\left[\Sigma_{\Lambda}, \delta \Lambda_{3}\right] Q_{\delta \Lambda_{2}} \Sigma_{\Lambda} Q_{\delta \Lambda_{1}}+\Sigma_{\Lambda} Q_{\delta \Lambda_{2}} \delta\left[\Sigma_{\Lambda}, \delta \Lambda_{3}\right] Q_{\delta \Lambda_{1}}\right\} \\
& =2 \operatorname{tr}\left\{\Sigma_{\Lambda} Q_{\delta \Lambda_{3}} \Sigma_{\Lambda} Q_{\delta \Lambda_{2}} \Sigma_{\Lambda} Q_{\delta \Lambda_{1}}+\Sigma_{\Lambda} Q_{\delta \Lambda_{2}} \Sigma_{\Lambda} Q_{\delta \Lambda_{3}} \Sigma_{\Lambda} Q_{\delta \Lambda_{1}}\right\}
\end{aligned}
$$

Similarly, since $\delta\left[\Sigma_{\Lambda}, \delta \Lambda\right]=\Sigma_{\Lambda} Q_{\delta \Lambda} \Sigma_{\Lambda}$, it can be shown that $J$ has continuous directional derivatives of any order in $\mathcal{L}$. Thus $J \in \mathcal{C}^{k}(\mathcal{L})$ for any $k \geq 0$.

\section{B. Proof of Proposition 5.1:}

Before proving that $\mathcal{L}_{\varphi}$ is bounded, we establish a preliminary lemma.

Lemma B.1: Let $Q_{\Lambda}$ be given by (27) and let $\varphi$ be given by (32). Given a sequence $\left\{\Lambda_{k}\right\}_{k \geq 0}$ with $\Lambda_{k} \in \operatorname{Range} \varphi$, if $\left\|\Lambda_{k}\right\| \rightarrow+\infty$ then $\left\|Q_{\Lambda_{k}}\right\| \rightarrow+\infty$.

Proof: The proof is divided into two steps.

Step 1: Consider a sequence $\left\{\Lambda_{k}\right\}_{k \geq 0}, \Lambda_{k} \in$ Range $\varphi$ such that $\left\|\Lambda_{k}\right\| \rightarrow+\infty$ as $k \rightarrow+\infty$. Since Range $\varphi=\operatorname{ker} \varphi^{\perp}$, the minimum singular value $\rho$ of the map $\varphi$ restricted to Range $\varphi$ is strictly positive. Accordingly

$$
\left\|\Pi_{B}^{\perp} \Lambda_{k} \Pi_{B}^{\perp}\right\| \geq \rho\left\|\Lambda_{k}\right\| \rightarrow+\infty .
$$

Step 2: It now remains to show that, if $\left\|\Pi_{B}^{\perp} \Lambda_{k} \Pi_{B}^{\perp}\right\| \rightarrow+\infty$ as $k$ tends to infinity, then also $\left\|Q_{\Lambda_{k}}\right\| \rightarrow+\infty$. We prove the following equivalent statement. Given a sequence $\left\{Q_{\Lambda_{k}}\right\}_{k \geq 0}$, if there exists $\alpha$ such that $\left\|Q_{\Lambda_{k}}\right\| \leq \alpha \forall k \geq 0$ then, there exists $\beta$ such that $\left\|\Pi_{B}^{\perp} \Lambda_{k} \Pi_{B}^{\perp}\right\| \leq \beta \forall k \geq 0$. Notice that equation

$$
Q_{\Lambda_{k}}=\Pi_{B}^{\perp} \Lambda_{k} \Pi_{B}^{\perp}-A^{*} \Pi_{B}^{\perp} \Lambda_{k} \Pi_{B}^{\perp} A
$$

is a discrete-time Lyapunov equation corresponding to the pair $\left(A^{*}, Q_{\Lambda_{k}}\right)$. Since $A$ is a stable matrix, we have

$$
\Pi_{B}^{\perp} \Lambda_{k} \Pi_{B}^{\perp}=\sum_{t=0}^{\infty}\left(A^{*}\right)^{t} Q_{\Lambda_{k}} A^{t} .
$$

Hence, $\forall k \geq 0$ we have

$$
\begin{aligned}
\left\|\Pi_{B}^{\perp} \Lambda_{k} \Pi_{B}^{\perp}\right\| & =\left\|\sum_{t=0}^{\infty}\left(A^{*}\right)^{t} Q_{\Lambda_{k}} A^{t}\right\| \leq \sum_{t=0}^{\infty}\left\|\left(A^{*}\right)^{t} Q_{\Lambda_{k}} A^{t}\right\| \\
& \leq \sum_{t=0}^{\infty}\left\|\left(A^{*}\right)^{t}\right\|\left\|Q_{\Lambda_{k}}\right\|\left\|A^{t}\right\| \\
& =\left(\sum_{t=0}^{\infty}\left\|A^{t}\right\|^{2}\right)\left\|Q_{\Lambda_{k}}\right\| \leq \gamma \alpha<+\infty
\end{aligned}
$$

where

$$
\gamma:=\sum_{t=0}^{\infty}\left\|A^{t}\right\|^{2} .
$$

The latter is a finite quantity since $A$ is a stable matrix.

We proceed to prove Proposition 5.1. By (30) and (34), it follows that $\mathcal{L}_{\varphi}$ is an open set. We know that each $\Lambda \in \mathcal{L}$ must satisfy the following inequality:

$$
2 Q_{\Lambda}>-\hat{\Sigma}^{-1} .
$$

Therefore

$$
\min \sigma\left(Q_{\Lambda}\right)>-\varepsilon^{2}
$$

where $\sigma(X)$ denotes the spectrum of the matrix $X$ and

$$
\varepsilon^{2}:=\frac{1}{2} \max \sigma\left(\hat{\Sigma}^{-1}\right) .
$$

We now show that a sequence $\left\{\Lambda_{k}\right\}_{k \geq 0}$, with $\Lambda_{k} \in \operatorname{Range} \varphi$, and $\left\|\Lambda_{k}\right\| \rightarrow+\infty$, cannot belong to $\mathcal{L}_{\varphi}$, i.e., $\mathcal{L}_{\varphi}$ is bounded. 
To this end, it suffices to show that the minimum eigenvalue of $Q_{\Lambda_{k}}$ tends to $-\infty$ so that, for $k$ large enough, $\Lambda_{k}$ does not satisfy (75). By Lemma B.1, $\left\|\Lambda_{k}\right\| \rightarrow+\infty$ implies that, as $k$ approaches infinity, $\left\|Q_{\Lambda_{k}}\right\| \rightarrow+\infty$. Hence

$$
\left\|\Sigma^{1 / 2} Q_{\Lambda_{k}} \Sigma^{1 / 2}\right\| \rightarrow+\infty
$$

for any given positive definite matrix $\Sigma>0$. In particular, if we choose $\Sigma \in$ Range $\Gamma$, since $Q_{\Lambda_{k}} \in$ Range $\Gamma^{\perp}$ (see Proposition 4.1), we have

$$
\left\langle Q_{\Lambda_{k}}, \Sigma\right\rangle=\operatorname{tr}\left(Q_{\Lambda_{k}} \Sigma\right)=\operatorname{tr}\left(\Sigma^{1 / 2} Q_{\Lambda_{k}} \Sigma^{1 / 2}\right)=0 .
$$

Since $\Sigma^{1 / 2} Q_{\Lambda_{k}} \Sigma^{1 / 2}$ is Hermitian, from (77) and (78) it follows that $\Sigma^{1 / 2} Q_{\Lambda_{k}} \Sigma^{1 / 2}$, and hence $Q_{\Lambda_{k}}$, have at least one eigenvalue tending to $-\infty$ as $k$ approaches infinity. In conclusion, there exist an integer $\bar{k}>0$ and an eigenvalue $\lambda_{k}$ of $Q_{\Lambda_{k}}$ such that

$$
\lambda_{k} \leq-\varepsilon^{2} \forall k>\bar{k} .
$$

Hence, in view of (75), $\Lambda_{k} \notin \mathcal{L}_{\varphi}, \forall k>\bar{k}$, and we may conclude that $\mathcal{L}_{\varphi}$ is a bounded set.

\section{Proof of Proposition 5.1:}

Since the minimum of $J$ exists and is unique, we investigate the global convergence of our Newton algorithm. To prove the convergence we need of the following result.

Proposition C.1: Consider a function $f: D \subset \mathbb{R}^{k} \rightarrow$ $\mathbb{R}$ twice differentiable on $D$ with $H_{x}$ the Hessian of $f$ at $x$. Suppose moreover that $f$ is strongly convex on a set $S \subset D$, i.e., there exists a constant $m>0$ such that $H_{x} \geq m I$ for $x \in S$, and $H_{x}$ is Lipschitz continuous on $S$. Let $\left\{x_{i}\right\} \in S$ be the sequence generated by the Newton algorithm. Under these assumptions, Newton's algorithm with backtracking converges globally. More specifically, $\left\{x_{i}\right\}$ decreases in linear way for a finite number of steps, and converges in a quadratic way to the minimum point after the linear stage.

Proof: See [34, 9.5.3, p. 488].

To prove the convergence of our algorithm, we proceed in the following manner: Identify a compact set $\tilde{S}$ such that $\Lambda_{i} \in \tilde{S}$ and prove that the second variation is coercive and Lipschitz continuous on $\tilde{S}$. We then apply Proposition C.1 in order to prove convergence.

Since $\Lambda_{0}=0$, we consider the set

$$
\tilde{S}:=\left\{\Lambda \in \mathcal{L}_{\varphi} \mid J(\Lambda) \leq J\left(\Lambda_{0}\right)=\frac{1}{2} \log \operatorname{det} \hat{\Sigma}\right\}
$$

which is compact (see Corollary 5.2). The presence of the backtracking stage in the algorithm guarantees that the sequence $J\left(\Lambda_{0}\right), J\left(\Lambda_{1}\right), \ldots$ is decreasing. Thus $\Lambda_{i} \in \tilde{S}, \forall i \geq 0$.

Proposition C.2: Consider the Hessian $\mathcal{H}_{\Lambda}$ defined in (41) and the associated quadratic form. The following facts hold:

1) As a quadratic form, $\mathcal{H}_{\Lambda}$ is coercive and bounded on $\tilde{S}$, namely there exist $m, M>0$ such that

$$
m\|\delta \Lambda\|^{2} \leq \mathcal{H}_{\Lambda}(\delta \Lambda, \delta \Lambda) \leq M\|\delta \Lambda\|^{2}, \forall \Lambda \in \tilde{S} .
$$

2) $\mathcal{H}_{\Lambda}$ is Lipschitz continuous on $\tilde{S}$.
Proof:

1. First, observe that $\tilde{S} \subset \mathcal{L}$. Since $\tilde{S}$ is a compact set, there exists $\varepsilon>0$ such that

$$
\Sigma_{\Lambda}=\left(\hat{\Sigma}+2 Q_{\Lambda}\right)^{-1} \geq \varepsilon I .
$$

Accordingly, for $\delta \Lambda \neq 0$,

$$
\begin{aligned}
\mathcal{H}_{\Lambda}(\delta \Lambda, \delta \Lambda) & =2 \operatorname{tr}\left[\Sigma_{\Lambda}^{1 / 2} Q_{\delta \Lambda} \Sigma_{\Lambda} Q_{\delta \Lambda} \Sigma_{\Lambda}^{1 / 2}\right] \\
& \geq 2 \varepsilon \operatorname{tr}\left[\Sigma_{\Lambda}^{1 / 2} Q_{\delta \Lambda} Q_{\delta \Lambda} \Sigma_{\Lambda}^{1 / 2}\right]=2 \varepsilon \operatorname{tr}\left[Q_{\delta \Lambda} \Sigma_{\Lambda} Q_{\delta \Lambda}\right] \\
& \geq 2 \varepsilon^{2} \operatorname{tr}\left[Q_{\delta \Lambda} Q_{\delta \Lambda}\right]=2 \varepsilon^{2} \gamma>0
\end{aligned}
$$

where $\gamma:=\operatorname{tr}\left[Q_{\delta \Lambda} Q_{\delta \Lambda}\right]>0$, since $Q_{\delta \Lambda}$ is not the zero matrix when $\delta \Lambda \neq 0$, as observed in the proof of Lemma 5.1. Since $J \in \mathcal{C}^{\infty}(\mathcal{L})$, it follows that $\mathcal{H}_{\Lambda}$ is continuous on the compact $\tilde{S}$ where it is also strictly positive definite. Hence, $\mathcal{H}_{\Lambda}$ is coercive and bounded on $\tilde{S}$.

2. $\mathcal{H}_{\Lambda} \in \mathcal{C}^{1}(\tilde{S})$ and $\left\|\mathcal{H}_{\Lambda}\right\| \leq M \forall \Lambda \in \tilde{S}$, therefore $\mathcal{H}_{\Lambda}$ is Lipschitz continuous on $\widetilde{\widetilde{S}}$.

Proposition C.3: The sequence $\left\{\Lambda_{i}\right\}_{i \geq 0}$ generated by the Newton algorithm of Section VI converges to the unique minimum point of $J$ in $\mathcal{L}_{\varphi}$.

Proof: Proposition C.1 applies to functions with domain contained in $\mathbb{R}^{k}, k \in \mathbb{N}$. The functional $J$ is defined over a subset of the linear space Range $\varphi$ which has finite dimension $d$ on $\mathbb{R}$. We define $x:=\operatorname{vect}(X)$ as the column vector (with $n^{2}$ entries) obtained by stacking the columns of $X$ one over the other, and we consider the following change of representation:

$$
\Lambda \mapsto \lambda=\operatorname{vect}(\Lambda) .
$$

Let be $D, S, f, H_{\lambda}$ the corresponding representation of $\mathcal{L}, \tilde{S}, J, \mathcal{H}_{\Lambda}$. It follows that:

- By Lemma 5.1, $f: D \subset \mathbb{R}^{d} \rightarrow \mathbb{R}:$ is twice differentiable on $S \subset D$

- By Proposition C.2 and since $\mathcal{H}_{\Lambda}(\cdot, \cdot)$ is a bilinear form, follows that $f$ is strongly convex on $S$ and $H_{\lambda}$ is Lipschitz continuous on $S$.

Therefore all the hypothesis of Proposition C.1 are satisfied and the conclusion follows.

\section{REFERENCES}

[1] J. Burg, D. Luenberger, and D. Wenger, "Estimation of structured covariance matrices," Proc. IEEE, vol. 70, no. 9, pp. 963-974, Sep. 1982.

[2] T. Georgiou, "Structured covariances and related approximation questions," in Directions in Mathematical Systems Theory and Optimization, A. Rantzer and C. Byrnes, Eds. Berlin, Germany: Springer, 2003, vol. 286, Lecture Notes in Control and Information Sciences, pp. $135-140$.

[3] C. I. Byrnes, T. Georgiou, and A. Lindquist, "A generalized entropy criterion for Nevanlinna-Pick interpolation with degree constraint: A convex optimization approach to certain problems in systems and control," IEEE Trans. Autom. Control, vol. 46, no. 6, pp. 822-839, Jun. 2001.

[4] C. I. Byrnes, T. Georgiou, and A. Lindquist, "A new approach to spectral estimation: A tunable high-resolution spectral estimator," IEEE Trans. Sig. Proc., vol. 49, no. 11, pp. 3189-3205, Nov. 2000.

[5] F. Ramponi, A. Ferrante, and M. Pavon, "A globally convergent matricial algorithm for multivariate spectral estimation," IEEE Trans. Autom. Control, vol. 54, no. 10, pp. 2376-2388, Oct. 2009.

[6] P. Stoica and R. Moses, Introduction to Spectral Analysis. New York: Prentice-Hall, 1997. 
[7] T. Georgiou, "Spectral estimation by selective harmonic amplification," IEEE Trans. Autom Control, vol. 46, no. 1, pp. 29-42, Jan. 2001.

[8] C. I. Byrnes, T. Georgiou, A. Lindquist, and A. Megretski, "Generalized interpolation in H-infinity with a complexity constraint," Trans. Amer. Math. Soc., vol. 358, pp. 965-987, Dec. 2004.

[9] C. I. Byrnes, S. Gusev, and A. Lindquist, "A convex optimization approach to the rational covariance extension problem," SIAM J. Control Opim., vol. 37, pp. 211-229, Oct. 1998.

[10] T. Georgiou and A. Lindquist, "Kullback-Leibler approximation of spectral density functions," IEEE Trans. Inform. Theory, vol. 49, no. 7, pp. 2910-2917, Nov. 2003.

[11] T. Georgiou and A. Lindquist, "Remarks on control design with degree constraint," IEEE Trans. Autom. Control, vol. 51, no. 7, pp. 1150-1156, Jul. 2006.

[12] T. Georgiou and A. Lindquist, "A convex optimization approach to ARMA modeling," IEEE Trans. Autom. Control, vol. 53, no. 5, pp. 1108-1119, Jun. 2008.

[13] A. Ferrante, M. Pavon, and F. Ramponi, "Hellinger vs. Kullback-Leibler multivariable spectrum approximation," IEEE Trans. Autom. Control, vol. 53, no. 4, pp. 954-967, May 2008.

[14] F. Carli, A. Ferrante, M. Pavon, and G. Picci, "A maximum entropy solution of the covariance extension problem for reciprocal processes," IEEE Trans. Autom. Control, vol. 56, no. 9, pp. 1999-2012, Sep. 2011.

[15] A. Blomqvist, A. Lindquist, and R. Nagamune, "Matrix-valued Nevanlinna-Pick interpolation with complexity constraint: An optimization approach," IEEE Trans. Autom. Control, vol. 48, no. 12, pp. 2172-2190, Dec. 2003.

[16] T. Georgiou, "The interpolation problem with a degree constraint," IEEE Trans. Autom. Control, vol. 44, no. 3, pp. 631-635, Mar. 1999.

[17] T. Georgiou, "Realization of power spectra from partial covariance sequences," IEEE Trans. Acoust., Speech, Signal Processing, vol. ASSP-35, no. 4, pp. 438-449, Apr. 1987.

[18] C. Byrnes, A. Lindquist, S. Gusev, and A. Matveev, "A complete parameterization of all positive rational extensions of a covariance sequence," IEEE Trans. Autom. Control, vol. 40, no. 11, pp. 1841-1857, Nov. 1995.

[19] C. I. Byrnes and A. Lindquist, "On the partial stochastic realization problem," IEEE Trans. Autom. Control, vol. 42, no. 8, pp. 1049-1070, Aug. 1997.

[20] T. Georgiou, "Spectral analysis based on the state covariance: The maximum entropy spectrum and linear fractional parameterization," IEEE Trans. Autom. Control, vol. 47, no. 11, pp. 1811-1823, Nov. 2002.

[21] T. Georgiou, "The structure of state covariances and its relation to the power spectrum of the input," IEEE Trans. Autom. Control, vol. 47, no. 7, pp. 1056-1066, Jul. 2002.

[22] T. Georgiou, "Solution of the general moment problem via a one-parameter imbedding," IEEE Trans. Autom. Control, vol. 50, no. 6, pp. 811-826, Jun. 2005.

[23] C. I. Byrnes and A. Lindquist, "Important moments in systems and control," SIAM J. Control Optim., vol. 47, pp. 2458-2469, Sep. 2008.

[24] C. I. Byrnes, P. Enqvist, and A. Lindquist, "Identifiability and wellposedness of shaping-filter parameterizations: A global analysis approach,” SIAM J. Control Optim., vol. 41, pp. 23-59, Mar. 2002.

[25] P. Enqvist and J. Karlsson, "Minimal Itakura-Saito distance and covariance interpolation," in Proc. 47th IEEE Conf. Decision Control, CDC'08, Sep./Nov. 2008, pp. 137-142.

[26] A. N. Amini, E. Ebbini, and T. Georgiou, "Noninvasive estimation of tissue temperature via high-resolution spectral analysis techniques," IEEE Trans. Biomed. Eng., vol. 52, no. 2, pp. 221-228, Feb. 2005.

[27] F. Ramponi, A. Ferrante, and M. Pavon, "On the well-posedness of multivariate spectrum approximation and convergence of high-resolution spectral estimators," Syst. Control Lett., vol. 59, pp. 167-172, Mar./Apr. 2010
[28] T. M. Cover and J. A. Thomas, Information Theory. New York: Wiley, 1991.

[29] M. A. Nielsen and I. L. Chuang, Quantum Computation and Quantum Information. Cambridge, U.K.: Cambridge Univ. Press, 2000.

[30] E. Jaynes, "On the rationale of maximum-entropy methods," Proc. IEEE, vol. 70, no. 9, pp. 939-952, Sep. 1982.

[31] A. Ferrante, M. Pavon, and F. Ramponi, "Further results on the ByrnesGeorgiou-Lindquist generalized moment problem," in Modeling, Estimation and Control: Festschrift in Honor of Giorgio Picci on the Occasion of His Sixty-Fifth Birthday, A. Chiuso, A. Ferrante, and S. Pinzoni, Eds. Berlin, Germany: Springer, 2007, pp. 73-83.

[32] M. Pavon and A. Ferrante, "On the Georgiou-Lindquist approach to constrained Kullback-Leibler approximation of spectral densities," IEEE Trans. Autom. Control, vol. 51, no. 4, pp. 639-644, Apr. 2006.

[33] A. Ferrante, M. Pavon, and F. Ramponi, "Constrained approximation in the Hellinger distance," in Proc. Eur. Control Conf. (ECC'07), Kos, Greece, Jul. 2007, pp. 322-327.

[34] S. Boyd and L. Vandenberghe, Convex Optimization. Cambridge, U.K.: Cambridge Univ. Press, 2004.

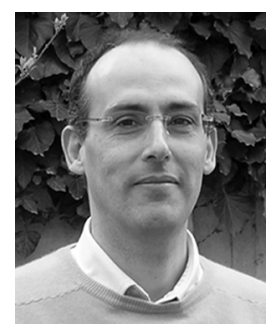

Augusto Ferrante was born in Piove di Sacco, Italy, on August 5, 1967. He received the "Laurea" degree (with high honors) in electrical engineering and the $\mathrm{Ph} . \mathrm{D}$. degree in control systems engineering from the University of Padova, Padova, Italy, in 1991 and 1995 , respectively.

He has been on the faculty of the Colleges of Engineering of the University of Udine and of the "Politecnico di Milano." He is presently Professor in the Department of Information Engineering of the University of Padova. His research interests are in the areas of linear systems, spectral estimation, optimal control and optimal filtering, quantum control, and stochastic realization.

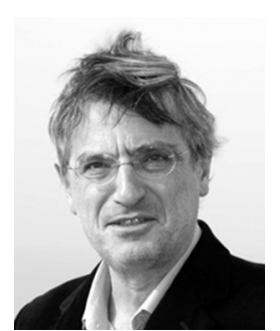

Michele Pavon was born in Venice, Italy, on October 12, 1950. He received the Laurea degree from the University of Padova, Padova, Italy, in 1974, and the Ph.D. degree from the University of Kentucky, Lexington, in 1979, both in mathematics.

After service in the Italian Army, he was on the research staff of LADSEB-CNR, Padova, for six years. Since July 1986, he has been a Professor at the College of Engineering, University of Padova. He has visited several institutions in Europe and Northern America. His present research interests include maximum entropy problems and quantum control.

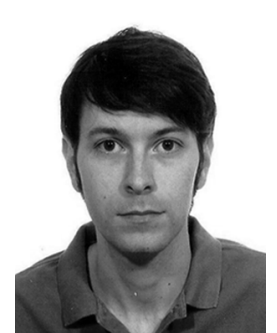

Mattia Zorzi was born in Padova, Italy, in January 1985. He received the B.S. degree (with high honors) in information engineering and the M.S. degree (with high honors) in automation engineering from University of Padova, Padova, Italy, in 2007 and 2009, respectively, where he is currently pursuing the Ph.D. degree.

His current research interests include spectral analysis and quantum systems estimation. 